\title{
Using Games in Community Disaster Prevention Exercises
}

\author{
Katsuya Yamori
}

\begin{abstract}
Currently, there is a growing interest in disaster prevention exercises such as games, debates, workshops, and "town walks" that involve the active participation of community members as an alternative to seminars in which disaster prevention experts simply share their knowledge and skills. We have developed a disaster prevention game called Crossroad for use in local disaster prevention programs. It is a group learning game, in which players read cards with a scenario written on each card. The scenarios were derived from extensive, focus-group interviews with disaster veterans of the Kobe Earthquake of 1995 that killed more than 6,400 people and described the dilemmas faced by these veterans. To deal with the dilemma, each player was required to make a "Yes" or No decision on two conflicting alternatives. We used Crossroad as a part of tsunami disaster prevention, community-building workshops for citizens of Kishiwada city in Osaka, Japan. The results suggested the importance of motivating local people to find a socially "viable" solution through their own strength, rather than forcing a community to accept a universally "correct" solution prescribed in advance by outsiders. It is concluded that the Crossroad game can be used effectively as a new communication medium to promote the active participation of community members.
\end{abstract}

Although it previously appeared in volume 21, number 4, 2012, this paper forms part of the Special Issue "Community-based Decision Making in Japan" edited by Norio Okada, Liping Fang, and D. Marc Kilgour.

K. Yamori $(\varangle)$

Disaster Prevention Research Institute, Kyoto University, Gokasho, Uji, Kyoto, Japan e-mail: momo-san@mx5.canvas.ne.jp 